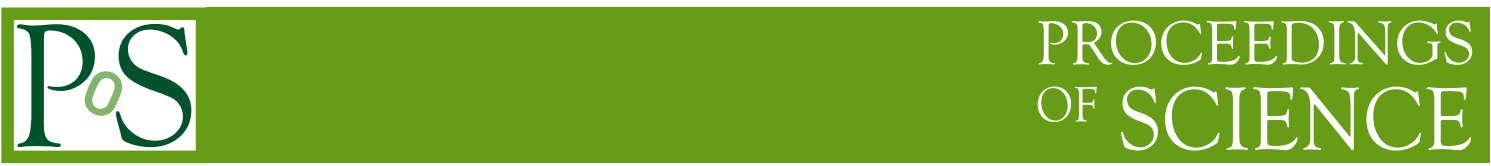

\title{
The Large-scale Structure of Warm Dark Matter
}

\author{
Katarina Markovič ${ }^{* \dagger}$ \\ University Observatory, Ludwig Maximillian University Munich \\ E-mail: markoviceusm. Imu.de
}

\begin{abstract}
Warm Dark Matter (WDM) is a generalisation of the standard Cold Dark Matter model in the sense that it does not assume dark matter particles to be absolutely cold. In the simplest models all dark matter is made of the same particles, which started out in thermal equilibrium and cooled to effectively become cold today. If such particles have masses of the order of a keV, they leave an imprint on the dark matter density field. At late times, the perturbations in the matter density field become non-linear. This means that they cannot be described perturbatively any longer. For this reason, N-body simulations are a good way to understand the formation of non-linear structure. Simulating WDM can be a challenge, because unlike CDM, it's relatively large thermal velocities can introduce unwanted Poisson noise on small scales. With better computing resources nowadays it has become possible to examine WDM cosmologies with simulations. This talk presented results of such simulations and discuss how to calculate non-linear corrections to the matter power spectrum in comparison to the halo model, which describes the matter density field today. It also discussed the possibility of constraining the dark matter particle mass using measurements of large scale structure, like cosmic shear or galaxy clustering.
\end{abstract}

Proceedings of the Corfu Summer Institute 2012

September 8-27, 2012

Corfu, Greece

\footnotetext{
* Speaker.

†This work was performed with support from the Trans-Regional Collaborative Research Center TRR 33 - "The Dark Universe" of the Deutsche Forschungsgemeinschaft (DFG).
} 


\section{Introduction}

The most concerning conflict with astronomical data of the $\Lambda \mathrm{CDM}$ model exists at the smallest cosmological scales in the distribution of structure in the universe (Simon \& Geha, 2007; de Blok, 2010). A possible reason for this issue may lay in the assumption that baryonic matter (luminous and dark) through its physical processes cannot significantly affect the DM overdensity field. If it can do so, it could erase power in the small scale dark matter overdensity field through feedback processes (e.g. van Daalen et al., 2011, and references therein). A further possibility would be warm dark matter (WDM), which can smooth the initial dark matter density field on small scales. An accurate description of small scales of cosmological structures is essential to utilise the full precision of future data for the extraction of the true cosmological model.

Both, WDM and neutrinos affects the structures in our universe by suppressing their formation. This is a result of both types of particles being relativistic for long enough to erase the perturbations in the cosmological energy density in a potentially observable way. This suppression is important on a range of scales that depends on the properties of the particles (Viel et al., 2005). In this talk, we have assumed for simplicity that our WDM particles are either sterile neutrinos or thermalised, supersymmetric gravitinos. Neutrinos make up a very small fraction of the total energy density and stay relativistic until the present day due to their small, sub-eV masses. This means that they affect the structure in our universe only mildly, but to relatively large scales. On the other hand, WDM consists, in its simplest form, of relatively heavy, $\mathrm{keV}$ particles, which become non-relativistic relatively early, but make up all of dark matter, therefore about $25 \%$ of the total energy density. This means that WDM suppresses the formation of small structure very strongly, but only up to relatively small scales, which are at present times described as very non-linear perturbations to the average background density.

Markovič et al. (2011) found discrepancies between the halofit (Smith et al., 2003) approach of calculating non-linear corrections to the linear power spectrum and the so-called halo model (Zentner, 2005) in the WDM scenario. They also found that it was difficult to determine how the halo bias and the halo mass function should be normalised. They suggested that dividing the matter field into a smooth and a clumped component might clarify this problem. They also found that the mass function derived from the suppressed linear matter power spectrum according to the Sheth \& Tormen (1999) formalism seemed to yield far too many haloes smaller than the free-streaming length of WDM, when comparing to N-body simulations of Zavala et al. (2008).

This talk has discussed the difficulties in modeling the highly non-linear structure in the WDM scenario by modifying the existing halo model of large-scale structure (sec.2) as well as running $\mathrm{N}$-body numerical simulations (sec.3), in order to investigate how the free-streaming of WDM, which smoothes out the sub- $0.1 \mathrm{Mpc}$ scales in the linear density field impacts the present day nonlinear density field. In other words, it is very difficult to describe the present day, non-linear density field analytically. This is not only because the full non-linear Einstein field equations have not yet been solved, but because even the approximation used in the linear regime (first order perturbation theory) no longer applies. This is the case already in the standard $\Lambda$ CDM model. However, many successful approaches have been developed over the past several decades. Unfortunately they become too inaccurate on the scales of interest, i.e. on the scales where WDM can account for the small scale issues of $\Lambda \mathrm{CDM}$. For this reason, we examine the possibility of improving these 
models.

\subsection{Free-streaming of DM Particles}

We encode the statistical properties of the matter density field with a power spectrum - a Fourier transformed 2-point correlation function of the field. In the case of WDM, the initial matter power spectrum emerging from radiation domination is modified by an additional transfer function due to free streaming. Viel et al. (2005) found this fitting function, but it can be calculated very accurately with a numerical Boltzmann equation solver code, like for example the CLAS S code (Blas et al., 2011). In the here described work, we use the fitting function with $v=1.12$ like in Viel et al. (2005) (the alternative is $v=1.2$ like in Bode et al. (2001)). Then the scale break parameter is:

$$
\alpha=0.049\left(\frac{m_{\mathrm{wdm}}}{1 \mathrm{keV}}\right)^{-1.11}\left(\frac{\Omega_{\mathrm{Wdm}}}{0.25}\right)^{0.11}\left(\frac{h}{0.7}\right)^{1.22}
$$

which is used in calculating the linear matter power spectrum by multiplying with the following warm transfer function:

$$
T_{\mathrm{wdm}}(k)=\left(1+(\alpha k)^{2 v}\right)^{-5 / v} \quad \text { and so } \quad P_{\mathrm{wdm}}(k)=P_{\mathrm{cdm}}(k) T_{\mathrm{wdm}}^{2}(k)
$$

(see Boyanovsky et al. (2008) for a calculation of the transfer function for a general initial thermal distribution of DM particles - cold WIMP dark matter, thermal fermionic or bosonic dark matter). The linear power spectrum, $P_{\mathrm{wdm}}(k)$, must then be normalised to ensure the value $\sigma_{8}^{2}$ at $k=$ $1 / 8 h \mathrm{Mpc}^{-1}$.

We now can plot the corresponding linear matter power spectra found using CLASS in fig. 1. The lightest WDM particle mass shown $(500 \mathrm{eV})$ causes the linear theory matter power spectrum to fall dramatically at a wavenumber significantly above $\sim 1 h \mathrm{Mpc}^{-1}$. The matter power spectrum of WDM starts to turn off well above the free-streaming scale, which changes the slope of the power spectrum to fall much more steeply than $n_{\text {eff }}=\log P(k) / \log k=-3$, which is the slope for standard, bottom-up structure formation.

In addition, we can define a corresponding mass found, on average, in a volume with such a radius or free-streaming:

$$
M_{\mathrm{fs}}=\frac{4 \pi \rho}{3}\left(\frac{\lambda_{\mathrm{fs}}}{2}\right)^{3}
$$

\section{Modifying the Halo Model}

It became clear in Markovič et al. (2011) that the halo model developed in the context of $\Lambda \mathrm{CDM}$ has some deficiencies in the $\Lambda \mathrm{WDM}$ model. For this reason we reexamined its ingredients: the mass function, the bias and the halo density profile in the new context. We describe an attempt at modifying these ingredients to better fit the assumptions we make about structure formation in the WDM scenario. The formalism was developed by Smith \& Markovič (2011) and was then employed in finding the non-linear corrections, weak lensing power spectra and Fisher matrices (FM) for the EUCLID survey. 


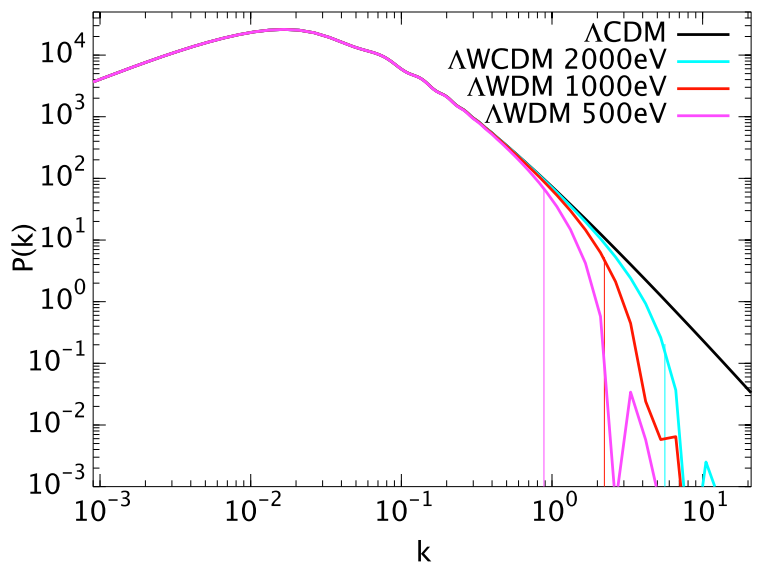

Figure 1: The linear matter power spectra for three different WDM models and standard CDM. The particle masses, $m_{\mathrm{wdm}} \in\{0.5,1.0,2.0\} \mathrm{keV}$ are color coded with magenta, red and cyan respectively. The vertical lines correspond to tenths of the free-streaming wavenumber, $k_{\mathrm{fs}} / 10$, for each model of WDM. The wiggles at $k \sim 0.1$ are due to baryonic acoustic oscillations and the peak at $k \sim 0.01$ corresponds to radiation-matter equality. On the left hand side of this peak, the slope corresponds to the fractal primordial power spectrum from inflation, where $P_{\delta}(k) \propto k$. The non-smoothness of the WDM curves on small scales is presumed to be numerical. This plot was obtained by running CLASS (Lesgourgues, 2011).

\subsection{Statistics of the split matter field}

If we describe the inhomogeneous density distribution of matter in the universe with the position dependent function $\rho(\vec{x})$, we can then attempt to split it up into a smoothly distributed component, $\rho_{\mathrm{S}}(\vec{x})$ and a component that is made up of many individual object - haloes, with particular density profiles $\rho_{\mathrm{h}}(r, M)$. These density profiles depend on the mass of the halo $M$ and since we assume these profiles to be spherically symmetrical, the distance from the halo centre, $r=\left|\vec{x}-\vec{x}_{0}\right|$. We can then sum over the individual objects to get the total density of the clumped component, $\rho_{\mathrm{c}}(\vec{x})$, and write the total density distribution in the universe as:

$$
\rho(\vec{x})=\rho_{\mathrm{S}}(\vec{x})+\sum_{i=1}^{N} M_{i} u_{\mathrm{h}}\left(\left|\vec{x}-\vec{x}_{0, i}\right|, M_{i}\right)
$$

where $u_{\mathrm{h}}(r, M)=\rho_{\mathrm{h}}(r, M) / M$ is the density profile normalised to the halo mass.

Averaging over the universe, we can define a background density, $\bar{\rho}$, which can also be split into a smooth and a clumped component:

$$
\langle\rho(\vec{x})\rangle=\left\langle\rho_{\mathrm{S}}(\vec{x})\right\rangle+\left\langle\rho_{\mathrm{c}}(\vec{x})\right\rangle=\bar{\rho}=\bar{\rho}_{\mathrm{S}}+\bar{\rho}_{\mathrm{C}}
$$

where we can define the fraction of matter contained within haloes as $f=\bar{\rho}_{\mathrm{C}} / \bar{\rho}$ and therefore also $(1-f)=\bar{\rho}_{\mathrm{S}} / \bar{\rho}$. In order to calculate the fraction of matter contained within haloes, we integrate over the differential mass function, $d n(M) / d M$ and cut the integral at a lower cut-off mass scale. This is reasonable because for now, we assume that no haloes exist with masses smaller than this cut-off, $M_{\text {cut }}$, which we equate to the free-streaming halo mass, $M_{\mathrm{fs}}$ - the mass of a halo with radius 
$r_{\mathrm{fs}} / 2$, a half of the free-streaming scale of WDM particles. Now we can re-write the fraction of matter contained within haloes as:

$$
f=\frac{1}{\bar{\rho}} \int_{M_{\mathrm{cut}}}^{\infty} d M M \frac{d n(M)}{d M}
$$

We wish to calculate the statistics of clustering in the form of the non-linear matter power spectrum, so we first start with the two-point correlation function of the matter density field, which we split into the two components:

$$
\langle\rho(\vec{x}) \rho(\vec{x}+\vec{r})\rangle=\left\langle\rho_{\mathrm{S}}(\vec{x}) \rho_{\mathrm{S}}(\vec{x}+\vec{r})\right\rangle+\left\langle\rho_{\mathrm{C}}(\vec{x}) \rho_{\mathrm{c}}(\vec{x}+\vec{r})\right\rangle+2\left\langle\rho_{\mathrm{C}}(\vec{x}) \rho_{\mathrm{S}}(\vec{x}+\vec{r})\right\rangle \quad,
$$

where we've set $\left\langle\rho_{\mathrm{S}}(\vec{x}) \rho_{\mathrm{c}}(\vec{x}+\vec{r})\right\rangle=\left\langle\rho_{\mathrm{c}}(\vec{x}) \rho_{\mathrm{S}}(\vec{x}+\vec{r})\right\rangle$, because we assume statistical homogeneity and isotropy in the standard cosmological model, meaning that the correlations in density between different points in the universe only depend on the distance between these points.

Now define, as it is common, the perturbation to the density field for either component, $\delta_{i}(\vec{x})$, where $i \in\{\mathrm{s}, \mathrm{c}\}$, by the equation:

$$
\rho_{i}(\vec{x}) \equiv \bar{\rho}_{i}\left(1+\delta_{i}(\vec{x})\right)
$$

and rewrite equation 2.4 , noting that by this definition, $\langle\delta(\vec{x})\rangle=0$ :

$$
\langle\rho(\vec{x}) \rho(\vec{x}+\vec{r})\rangle=\bar{\rho}_{\mathrm{S}}^{2}\left(1+\xi_{\mathrm{SS}}(r)\right)+\bar{\rho}_{\mathrm{c}}^{2}\left(1+\xi_{\mathrm{cc}}(r)\right)+2 \bar{\rho}_{\mathrm{c}} \bar{\rho}_{\mathrm{S}}\left(1+\xi_{\mathrm{cS}}(r)\right)
$$

where we've replaced in the definition of a correlation function as:

$$
\xi_{i j}(|\vec{r}|) \equiv \xi_{i j}(r) \equiv\left\langle\delta_{i}(\vec{x}) \delta_{j}(\vec{x}+\vec{r})\right\rangle
$$

Note also that the total perturbation is

$$
\delta(\vec{x})=\frac{\rho(\vec{x})-\bar{\rho}}{\bar{\rho}}=\frac{\bar{\rho}_{\mathrm{S}} \delta_{\mathrm{S}}(\vec{x})+\bar{\rho}_{\mathrm{c}} \delta_{\mathrm{c}}(\vec{x})}{\bar{\rho}}=(1-f) \delta_{s}(\vec{x})+f \delta_{\mathrm{C}}(\vec{x})
$$

Using the definition of the fraction of clumped matter, we can now rearrange equation 2.6 to obtain:

$$
\xi(r)=(1-f)^{2} \xi_{\mathrm{sS}}(r)+2 f(1-f) \xi_{\mathrm{sc}}(r)+f^{2} \xi_{\mathrm{cc}}(r) .
$$

So the total correlation function of the over-density field is just a sum of the correlation functions and the cross-correlation of the smooth and clumped fields, all weighed by the fraction of matter contained within haloes, $f$. See Smith \& Markovič (2011) on how to calculate these terms.

\subsubsection{Bias of the smooth, linear-like matter field}

We now need to find the bias factors for the smooth component. If mass is to be conserved, knowing this halo bias, we can immediately derive the bias of the smooth component. In other words, fully knowing how the underlying total dark matter distribution determines the distribution of haloes, we can fully understand the density distribution of the smooth component without much further effort. See Smith \& Markovič (2011) for a detailed derivation of:

$$
b_{\mathrm{S}, 1}=\frac{1-f b_{1}^{\mathrm{eff}}}{1-f} \quad \text { and } \quad b_{\mathrm{S}, i}=-\frac{f b_{\mathrm{c}, i}^{\mathrm{eff}}}{1-f} \quad(i \neq 1)
$$




\subsubsection{The total power spectrum}

Now to obtain power spectra, we must Fourier transform and so the weighted contributions from the 3 separate correlation combinations become:

$$
P(k)=(1-f)^{2} P_{\mathrm{SS}}(k)+2(1-f) f P_{\mathrm{Sc}}(k)+f^{2} P_{\mathrm{cc}}(k),
$$

where

$$
\begin{aligned}
P_{\mathrm{SS}}(k) & =b_{\mathrm{S}, 1}^{2} P_{R}(k), \\
P_{\mathrm{Sc}}(k) & =\frac{b_{\mathrm{S}, 1} P_{R}(k)}{\bar{\rho}_{\mathrm{c}}} \int_{M_{\mathrm{cut}}}^{\infty} d M M b_{1}(M) \frac{d n(M)}{d M} \tilde{u}(k, M) \\
P_{\mathrm{cc}}(k) & =\frac{P_{R}(k)}{\bar{\rho}_{\mathrm{c}}^{2}}\left[\int_{M_{\mathrm{cut}}}^{\infty} d M M b_{1}(M) \frac{d n(M)}{d M} \tilde{u}(k, M)\right]^{2} \\
& +\frac{1}{\bar{\rho}_{\mathrm{c}}^{2}} \int_{M_{\mathrm{cut}}}^{\infty} d M \frac{d n(M)}{d M} M^{2} \tilde{u}^{2}(k, M),
\end{aligned}
$$

where $\tilde{u}(k, M)$ is the Fourier-transformed, mass-normalised halo density profile and where we may approximate the smoothed non-linear power spectrum, $P_{R}(k)$ with the WDM suppressed linear power spectrum 1.2. The two terms in the expression for $P_{\mathrm{cc}}$ corresppond to the standard two- and one-halo i.e. Poisson and Clustering terms.

\subsection{Modified halo mass functions}

There seems to be an excess in the number of low mass haloes obtained if the standard ShethTormen approach is used for WDM compared to simulations (Zavala et al., 2009; Polisensky \& Ricotti, 2010). Moreover, considering that the initial density field becomes strongly smoothed on small scales by WDM free-steaming, it may be argued that small haloes should not form on their own and may perhaps only be able to exist within much larger haloes (Bode et al., 2001; Wang \& White, 2007). This comes from considering peak theory of structure formation. Namely, if peaks corresponding to short wavenumbers in Fourier space become suppressed in amplitude, they should only end up as haloes if they are initially in a high density environment, meaning that they overlap with peaks corresponding to large Fourier wavenumbers, which still can have large amplitudes.

For this reason, we have introduced an arguably ad-hoc modification to the mass functions, which suppressed the number of the very smallest haloes to zero. We multiply the calculated Sheth-Tormen mass functions, $d n / d M$, from our WDM-suppressed linear matter power spectra by a smoothed step function that depends on the halo mass rescaled by the free-streaming halo mass, $M_{\mathrm{fs}}$ as defined above:

$$
\frac{d \tilde{n}_{\mathrm{Wdm}}}{d \log M}=\frac{1}{2}\left\{1+\operatorname{erf}\left[\frac{\log _{10}\left(M / M_{\mathrm{fs}}\right)}{\sigma_{\log M}}\right]\right\} \frac{d n_{\mathrm{Wdm}}}{d \log M}
$$

where we have chosen the smoothing parameter for the step function $\sigma_{\log M}=0.5$. Were we to chose this smoothing parameter to equal zero, our smooth step function would become the Heaviside step function. The free-streaming mass on the other hand decides the halo mass at which the suppression is applied. For CDM, $M_{\mathrm{fs}} \rightarrow 0$ and so, $d \tilde{n} \rightarrow d n, \operatorname{since} \operatorname{erf}(x) \rightarrow 1$ for $x \rightarrow \infty$. 


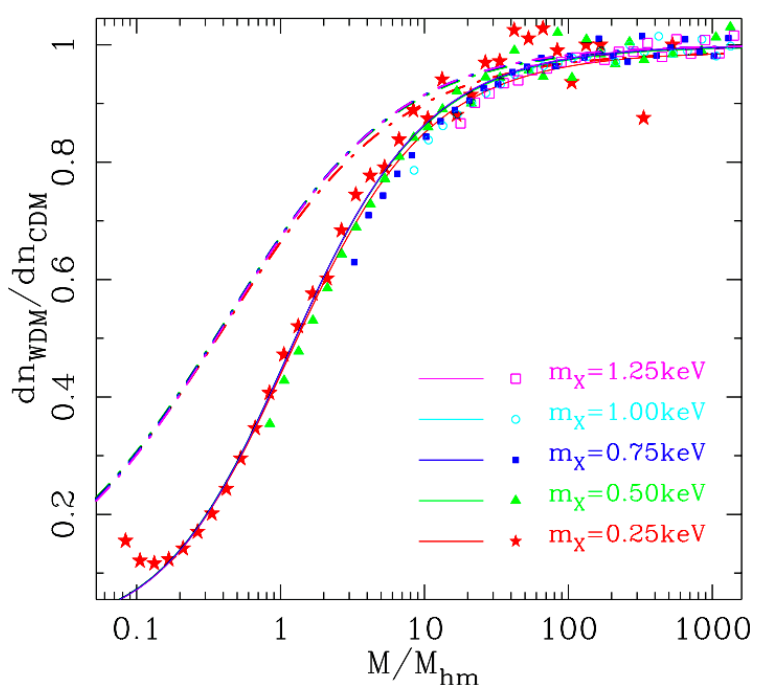

Figure 2: Mass function ratios comparing WDM and CDM halo mass distribution are shown here for 5 different thermal relic WDM particle masses (different colors). The halo masses are rescaled with respect to the half-mode mass. The dot-dashed lines are the theoretically calculated mass functions using the Sheth-Tormen formalism starting from a WDM-suppressed linear matter power spectrum. The star, triangle, filled square, circle and empty square shaped points are the results from N-body simulations. For this, halo finder code had to be run on the simulation results by the paper authors. The solid lines correspond to the universal fit. It can be seen here that the shape of the suppression of the mass functions is independent of the WDM model. The WDM dependence comes in only via the halo mass rescaling, which is to be expected, because the rescaling is calculated from the behaviour of the mass function. FIGURE SOURCE: Schneider et al. (2012)

More recently (Schneider et al., 2012) examined this model in comparison to N-body simulations. Fig. 2 shows their result, plotting the ratio of the WDM and CDM mass functions. They rescaled the halo masses with respect to the so-called "half-mode mass", $M_{\mathrm{hm}} \approx 2.7 \times 10^{3} M_{\mathrm{fs}}{ }^{1}$, rather than the free-streaming mass as above. They find the simple fitting formula:

$$
\frac{d \tilde{n}_{\mathrm{wdm}}}{d n_{\mathrm{wdm}}}=\left(1+\frac{M_{\mathrm{hm}}}{M}\right)^{-\alpha},
$$

to match their simulation results well without the need to apply an artificial step function. The single fitting parameter, $\alpha=0.6$ was able to match the simulations with less than 5\% root-meansquare error. Dunstan et al. (2011) find very similar results.

\subsection{Forecasts for non-linear measurements}

We now plot only the weighted sum of these components using the mass function from eq. 2.13 , i.e. the total non-linear matter power spectrum for different WDM as well as the CDM model in fig. 3. We also plot the ratio of the WDM and CDM non-linear power. We plot these together the analogous linear matter power spectra and see that through non-linear collapse power is regenerated. This matches the conclusions of Markovič et al. (2011). We also compare this new modified halo model to the HALOFIT. We can see that the general trend is similar, but in the halo

\footnotetext{
${ }^{1}$ The half-mode mass is defined to be the mass corresponding to he scale where the WDM linear transfer function in eq. 1.2 drops to a value of 0.5 .
} 


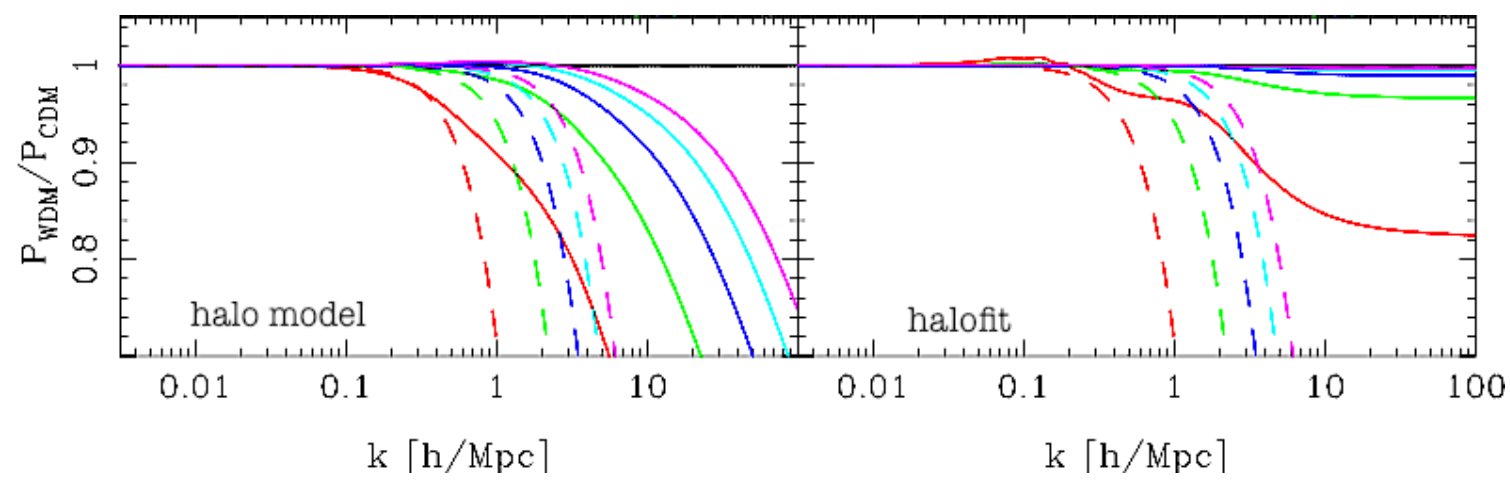

Figure 3: The ratios between the WDM and CDM non-linear (solid) as well as linear (dashed) matter power spectra for different WDM models are plotted, where the particle masses for a thermal relic, $m_{\mathrm{wdm}} \in$ $\{0.25,0.5,0.75,1.0,1.25\} \mathrm{keV}$. The power spectra are calculated assuming the new, modified halo model described here, without modifications of Schneider et al. (2012). On the right panel are the ratios plotted using the HALOF I prescription for comparison. FIGURE SOURCE: Smith \& Markovič (2011)

model the suppression of the power spectrum is significantly stronger. For example, in the most extreme model, where $m_{\mathrm{wdm}}=0.25 \mathrm{keV}$, the suppression at the wavenumber, $k \sim 10 \mathrm{hMpc}^{-1}$ is greater than $30 \%$. On the other hand, in the H A L OF I T, the suppression in this same model saturates already at scales $k \sim 10 \mathrm{hMpc}^{-1}$ to a maximum of $\sim 18 \%$.

\section{Cosmological Simulations}

Viel et al. (2012) ran cosmological N-body simulations, where we set up the initial conditions to reflect the small-scale suppressed power spectra. The effect of baryonic cooling on the underlying dark matter distribution are briefly considered. We give a fitting function to the simulation results for the non-linear corrections to the WDM suppressed linear matter power spectrum. We compare all the approaches: the classic HALOF I T, the new halo model and the fit to simulations.

We used the TreePM N-body code GADGET-2 to run mostly pure dark matter simulations. For a few runs we also added baryonic particles into our simulations for which GADGET-2 uses the SPH (Lagrangian) approach (Springel, 2005). In our runs we vary the sizes of our cosmological volumes to account for any large scale errors and more importantly to test the effects of resolution. The issue of spurious halo formation on small scales has been a long standing one and we want to make sure we are not plagued by such effects. The gravitational softening is set to be $1 / 40$ th of the mean linear inter-particle separation and is kept fixed in comoving units.

\subsection{The non-linear power spectrum from simulations}

We ran several simulations that varied in their volume and WDM model mainly, but we also ran one simulation that includes baryonic cooling. In fig. 4 we show slices from two of the simulations, which are projections of $2.5 \mathrm{Mpc}$-thick slices through the $25^{3} \mathrm{Mpc}^{3}$ simulation volume for the CDM and the $1 \mathrm{keV}$ WDM model. In this simulation run, we set the number of simulation particles to $N=512^{2}$ and the cosmological box side to $L=25 \mathrm{Mpc}$, which gives simulations particles the masses of $8.7 \times 10^{6} M_{\odot} h^{-1}$, which is assumed to be sufficiently below the free-streaming mass. 

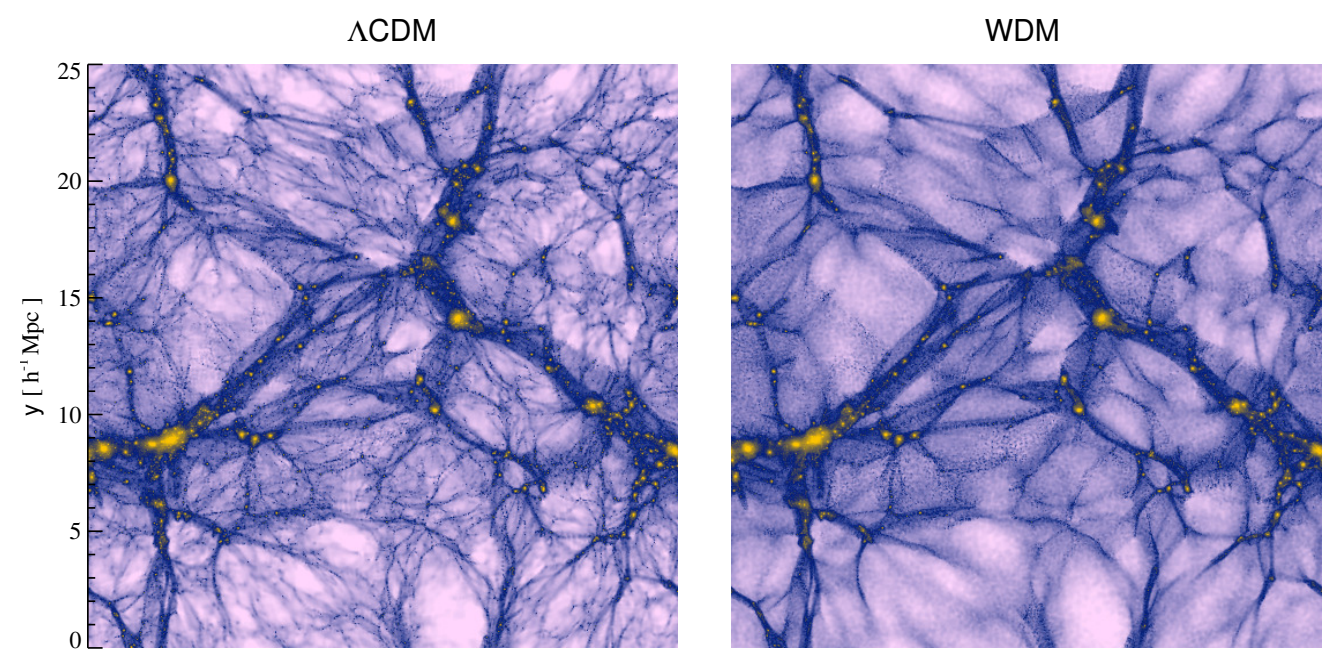

Figure 4: Here are plotted $2.5 \mathrm{Mpc}$-thick slices of the simulation at redshift $z=0$ for $\Lambda \mathrm{CDM}$ (left) and $m_{\mathrm{wdm}}=1 \mathrm{keV} \Lambda \mathrm{WDM}$ (right). The clustering is indistinguishable on scales $k<10 \mathrm{Mpc}$, but at $z=2,5$ the WDM model is smoothed by WDM free-streaming, which results in a suppression in the power spectrum of about $5 \%$ and $25 \%$ (respectively) at $k \sim 10 \mathrm{hMpc}^{-1}$. FIGURE SOURCE: Viel et al. (2012)

The shot-noise power due to the finiteness of our simulation particles was subtracted from all the power spectrum estimates made. The shot-noise power becomes comparable to the real, physical power at $z=0$ at $k \sim 150 \mathrm{hppc}^{-1}$ for the simulation box side of $L=100 \mathrm{Mpc}$. For the simulation with $L=25 \mathrm{Mpc}$ in the WDM model with $m_{\mathrm{wdm}}=1 \mathrm{keV}$ the matter power is always larger than the shot-noise power for $z<10$ and for $k<20 \mathrm{hMpc}^{-1}$.

It was checked in Viel et al. (2012) that increasing the Particle-Mesh grid by a factor three impacts the matter power spectrum on scales, $k<100 \mathrm{hMpc}^{-1}$ negligibly. In this work a higher resolution simulation was run by increasing the number of particles in a box with $L=25 \mathrm{Mpc}$ to $N=640^{3}$ and yielded a less than $1 \%$ difference on scales, $k<100 \mathrm{Mpc}$. This is an indicator that spurious halo formation plagues the non-linear matter power spectrum on relevant scales minimally.

\subsection{The fitting formula}

In analogy to the linear fitting formula in eq. 1.2, which defines a scale-break, $\alpha$, we found the following fitting function to the results of our dark-matter only simulations:

$$
\begin{aligned}
T_{\mathrm{nl}}^{2}(k) & \equiv P_{\mathrm{wdm}}(k) / P_{\mathrm{cdm}}(k)=\left(1+(\alpha k)^{v l}\right)^{-s / v} \\
\alpha\left(m_{\mathrm{wdm}}, z\right) & =0.0476\left(\frac{1 \mathrm{keV}}{m_{\mathrm{wdm}}}\right)^{1.85}\left(\frac{1+z}{2}\right)^{1.3},
\end{aligned}
$$

where $v=3, l=0.6$ and $s=0.4$ are the fitting parameters. This function was applied by first calculating the non-linear matter power spectrum using $\Lambda$ CDM parameter values in the HA L OF I T and then multiplying with the square of the WDM "transfer function".

At $z=0$ the suppression only remains significant for the extreme WDM models, which have been ruled out by Ly $\alpha$ forest data (Viel et al., 2005; Seljak et al., 2006). 

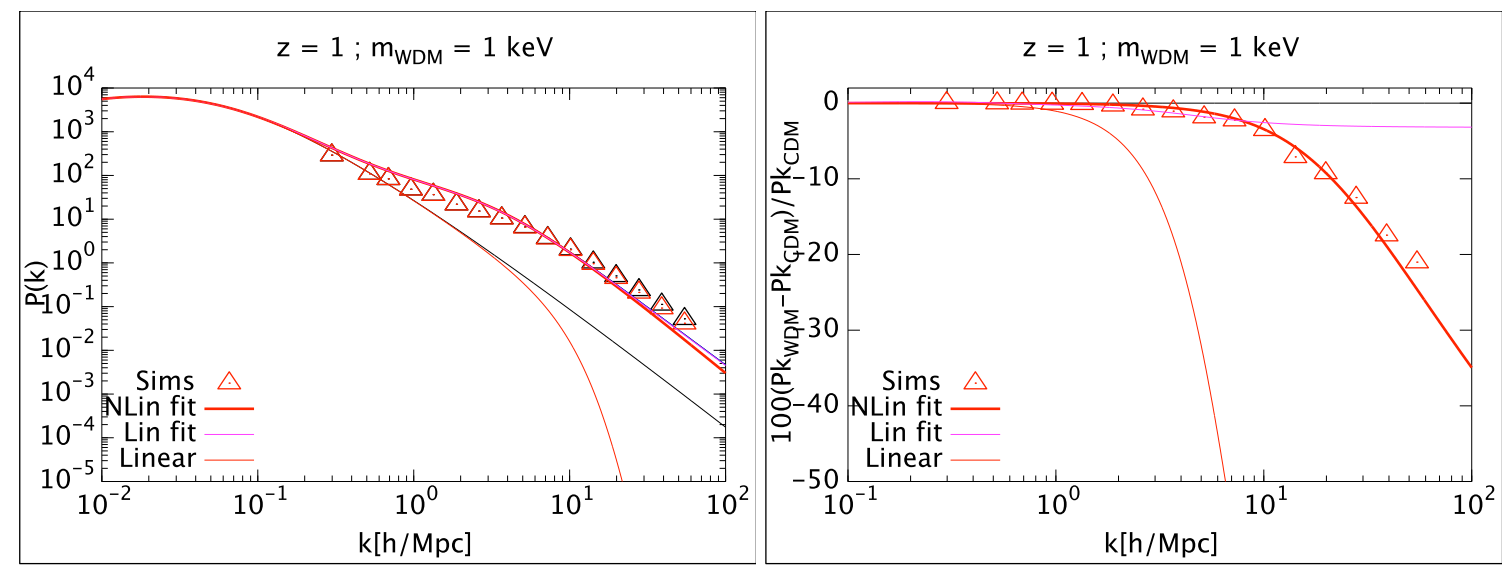

Figure 5: The comparison of the non-linear fits to simulations at redshift $z=1$ for WDM particles with mass $m_{\mathrm{wdm}}=1 \mathrm{keV}$. The triangles represent the power spectra and fractional differences calculated from DM-only simulations. The solid lines are the corresponding analytical fits.

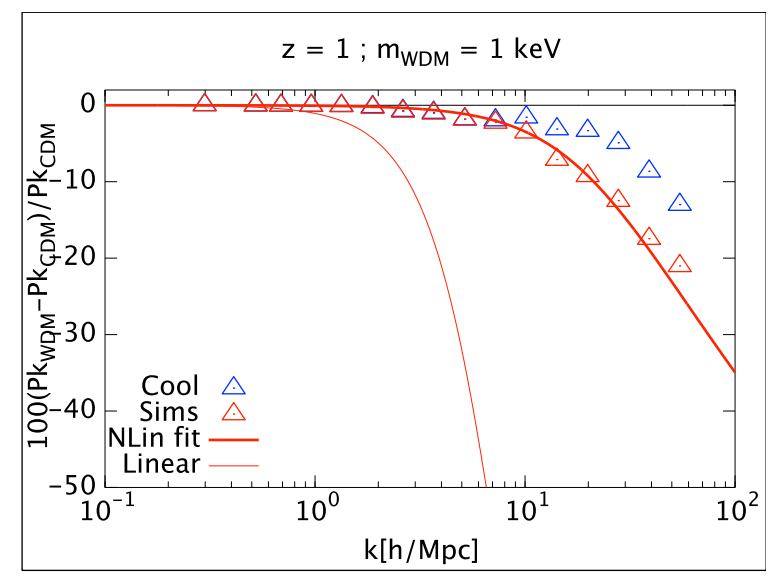

Figure 6: Here we plot the percentage differences between the WDM and CDM power spectra. We have included the power spectra runs that include baryonic cooling due to $\mathrm{H}$ and $\mathrm{He}$ (blue). The prescription used for the cooling processes is called "quick Ly $\alpha$ ".

\subsection{Comparison with HALOFIT and WDM halo model}

Here, we compare the final matter power spectra found from simulations with those from the WDM halo model above and plot the comparison in fig. 5.

As has been shown by many authors (e.g. van Daalen et al., 2011; Semboloni et al., 2011), baryons, making up $17 \%$ of the total matter density, affect the distribution of dark matter on small scales significantly. We do briefly discuss one simulation run by Viel et al. (2012), which includes a prescription for radiative cooling and heating. In this simulation all the cooling comes from hydrogen and helium. Metal cooling is omitted for simplicity. Secondly, the feedback from supernovae and AGN is also omitted in this particular run. The prescription for modeling the cooling and the star fromation criterion are described in more detail in Viel et al. (2004) and is called "quick Ly $\alpha$ ", since it can be used well for quantitative description of the Ly $\alpha$ forest.

We plot in 6, the resulting percentage difference between a $1 \mathrm{keV}$ WDM and CDM non-linear 
matter power spectrum, where both come from simulations that include cooling and heating processes from the ultraviolet background and a simple star formation criterion mentioned above. This simulation was stopped at $z=1.2$ due to limited computational resources and is therefore plotted at this redshift. It can be seen on 6 that the inclusion of baryonic processes can have a very significant effect on the suppression signal from WDM. It seems likely that some baryonic processes become more efficient in a collapsing overdensity field that has been smoothed. Because the baryonic processes affect the power on small scales, this can erase the suppression from WDM, which is relevant on similar scales.

\section{Future measurements}

So far we have discussed models in which all dark matter is made up of thermal relic particles with $\mathrm{keV}$ masses. In other words, all dark matter is warm. However a more realistic picture would be to take into account a component of the dark matter density we know exists - massive neutrinos. Massive neutrinos have sub-eV masses. In fact most recent constraints from cosmology put the sum of the 3 neutrino masses of the electron, $\tau$ and $\mu$ neutrinos to be somewhere $M_{v}<0.5 \mathrm{eV}$. For this reason we classify neutrinos as HDM. In fact such a mixed dark matter model can contain many different species, with different temperatures, interactions or decay mechanisms. However such additional properties of the dark matter density field necessarily introduce further parameters into the model. Until such addition of parameters is justified by a physical theory, let us take the next simplest case from what we have discussed now and examine the combined effect of WDM and massive neutrino free-streaming in the initial density field on the formation of cosmological structure.

In particular, since the detectability of the WDM signature is still uncertain and since detecting the neutrino mass is within the possibility of upcoming data, we wish to ensure that there will be no degeneracy between the two parameters: the sum of neutrino masses, $M_{v}$ and the WDM particle mass, $m_{\mathrm{wdm}}$.

\subsection{The linear regime suppression in $v \Lambda \mathrm{WDM}$}

We again use the CLASS Boltzmann solver code to find the transfer functions that account for the suppression of the primordial power spectra.

We plot the resulting linear matter power spectra for the models: $\Lambda \mathrm{CDM}, v \Lambda \mathrm{CDM}, \Lambda \mathrm{WDM}$ and $v \Lambda \mathrm{WDM}$, in the left panel of 7 . The model used for WDM is that where the particle mass is $m_{\mathrm{wdm}}=1 \mathrm{keV}$, for neutrinos we use such parameter values as to get the energy density fraction for neutrinos to be $\Omega_{v}=0.025$. This density fraction corresponds to the sum of neutrino masses of $M_{v} \approx 2.3 h^{2} \mathrm{eV}$ (Bird et al., 2012). The mentioned artefacts can be seen at scales of $k \sim 5 h \mathrm{Mpc}^{-1}$. In this figure, the solid lines are the power spectra in both $\Lambda$ WDM models - one with and one with no massive neutrinos. The dotted lines are models with regular CDM. What we note in this plot is that the suppression of the linear scales is much stronger when considering WDM, but it reaches much larger scales (smaller $k$ ) if the free-streaming of massive neutrinos is accounted for. Including both, massive neutrinos and WDM results in a twice suppressed power. The two suppression occur at two different scales with two different amplitudes. The reason why the suppression is so much stronger in the $\Lambda \mathrm{WDM}$ model is because in this case all of DM is warm, whereas in the $v \Lambda \mathrm{CDM}$ 

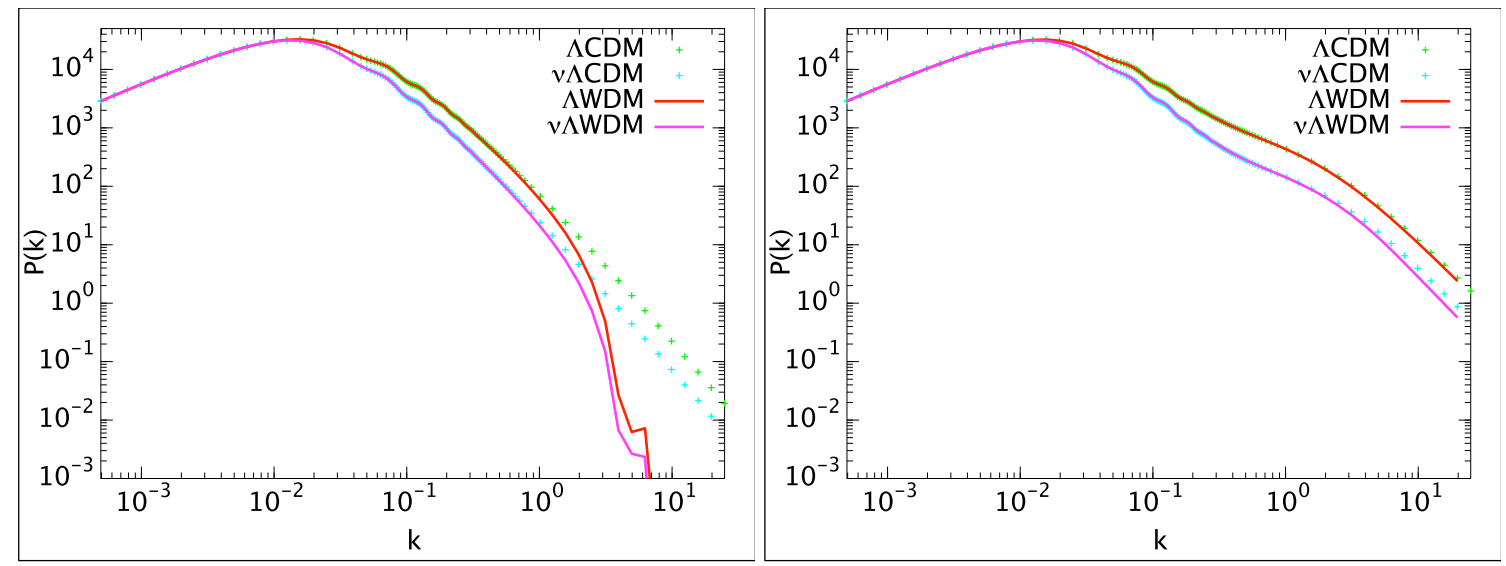

Figure 7: We plot the power spectra showing the suppression due to the free-streaming of massive neutrinos as well as WDM thermal relic particles in the early universe. We use $m_{\mathrm{wdm}}=1 \mathrm{keV}$ and $\Omega_{v}=0.025$ for illustration. In the left panel we plot the linear matter power spectra. The dotted lines are the CDM models and the solid lines correspond to WDM. In each of the two sets of lines, the line with less power has been suppressed by the free-streaming of neutrinos. Note also that on very small scales in the WDM suppressed functions become jagged. We suspect this is a numerical artefact. We have checked and such "wiggles" appear in results obtained with several Boltzmann-solver codes: CLAS S, CAMB and CMBF A T.

case, only a tiny fraction of dark matter is mase up of massive neutrinos. The idea of massive neutrinos making up a significant part of dark matter density was rejected long ago for this reason, as the suppression would be too strong for these large scales. If neutrinos dominated the matter energy density, there would be virtually no power in the power spectrum on scales $k>0.1 \mathrm{hMpc}^{-1}$ and therefore we would observe no structures in the universe.

\subsection{Nonlinear corrections in $v \Lambda \mathrm{WDM}$}

We use the HALOFIT prescription as is given in the CLASS code. There exists a correction to the pure, basic $\Lambda$ CDM HALOF I T, which takes $f_{v}=\Omega_{v} / \Omega_{\mathrm{m}}$, the fraction of the cosmic matter density made of neutrinos, as a parameter. This correction is based on the N-body simulations run by Bird et al. (2012) and means adding an additional neutrino-mass-dependent term to the HALOF I T dimenstionless power spectrum.

We plot the resulting power spectra in the right panel of fig. 7. The color scheme matches that on the left, where we plotted the linear matter power spectra described in the previous section. It is immediately obvious that as before, the non-linear correction erases a lot of the suppression from the linear regime. As we have discussed in the previous chapters, this is likely to be a consequence of smooth accretion and general correlation of the small scale modes by gravitational collapse. On the other hand, the suppression due to neutrinos doesn't seem to be much affected by this. The reason is that whereas the scale of suppression due to WDM is smaller, the scale of the suppression due to neutrinos is larger than the non-linear scale, i.e. $k_{\mathrm{wdm}}>k_{\mathrm{nl}}>k_{v}$.

\section{Conclusions}

The three approaches in this talk to calculating the non-linear power spectrum, which describes 

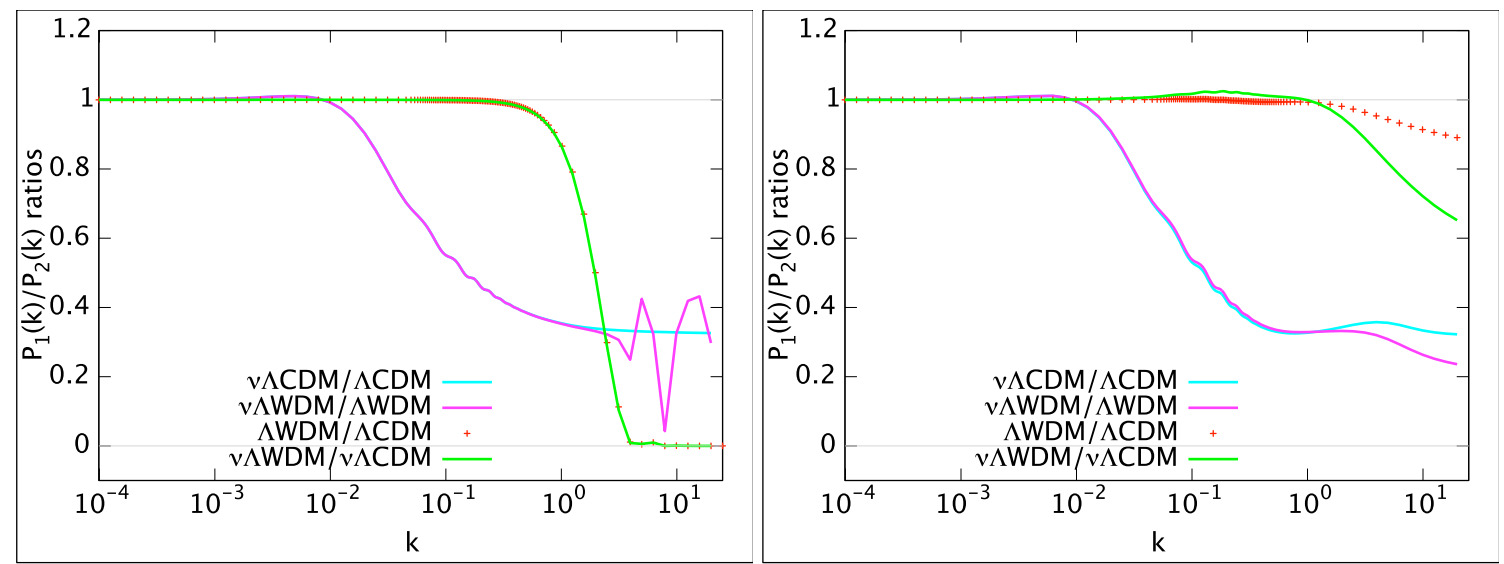

Figure 8: We plot the ratios of matter power spectra suppressed on small scales by the free-streaming of non-cold dark matter species. We find ratios of the expected $\Lambda \mathrm{WDM}, v \Lambda \mathrm{CDM}, v \Lambda \mathrm{WDM}$ or the standard $\Lambda \mathrm{CDM}$ power spectra. The the blue and magenta lines are the scenarios that highlight the suppression due to massive neutrinos, the green and dotted red highlight the suppression due to WDM. In the left panel we plot only ratios of linear, on the right we plot only spectra. The linear spectra were calculates using the CLASS Boltzmann solver. For non-linear corrections we used the HALOFIT prescription within CLASS. The used non-linear prescription includes a correction for the presence of massive neutrinos (Bird et al., 2012).

the statistics of the present-day density field, have been the halo model, the related HALOF I prescription and the full numerical solving of the Newtonian dynamics: N-body simulations. We have attempted to modify the first two in such a way as to be compatible with the third.

The halo model modifications we made were at first not calibrated against $\mathrm{N}$-body simulations and so they slightly overestimated the suppression in the non-linear power spectrum in the $\Lambda \mathrm{WDM}$ scenario. This was done subsequently by other authors, who have suggested a useful prescription for the halo mass function in the WDM scenario (Schneider et al., 2012).

Our modification to the HALOFIT was in the form of an additional WDM transfer function, which we fit to the results of our N-body simulations. We ran these simulations using a nonmodified version of the GADGET-2 code (Springel, 2005). To include the effects of WDM, we created initial conditions for the simulation, whose statistics were described by a suppressed linear matter power spectrum. We then ran the unmodified GADGET-2 code, since by the time of gravitational collapse, the WDM particles have lost most of their thermal energy due to the expansion of the universe. Their velocities have become low enough to be negligible for the purposes of finding the matter power spectrum on scales larger than the very centres of our haloes. The fitting function resulting from our simulations is useful for calculating theoretical WDM power spectra in order to compare them to data of cosmic structure.

We have touched upon the subject of baryonic physics, modeled by hydrodynamical simulations. We ran one simulation that included some baryonic cooling as well as star formation, which has indicated that such effects affect the non-linear matter power spectrum (via gravitational coupling) similarly and on similar scales to WDM. Even though such numerical prescriptions are yet uncertain, it is clear that before a measurement or constraint on the WDM mass is made, one must be familiar with the effects of baryonic physics. A potential solution to this important degeneracy may be the examination of the evolution of these effects. Whereas the WDM suppression increases 
with increasing redshift, the effect of baryons may have an entirely different signature in redshift. This would be interesting to model using numerical methods, but it would be a large undertaking as it would require extensive computational resources.

The small scales of cosmological structures are shaped by several different physical processes. Their exact effects are difficult to predict and the field of cosmology concerned with them is relatively young. Besides baryonic effects a WDM would impact the small scales by changing the initial conditions. The presence of such dark matter could elegantly explain some of the conflicts with data that arise when assuming that all dark matter is cold. Because the upcoming cosmological surveys will be capable of impressive precision, they will be able to map out the 3D dark matter density field to a resolution achieved never before. This will give us a chance to test the WDM model by applying the non-linear prescriptions developed in the work described by this talk.

\section{References}

Bird S., Viel M. \& Haehnelt M.G. (Mar. 2012). Massive neutrinos and the non-linear matter power spectrum. In MNRAS, vol. 420, pp. 2551.

Blas D., Lesgourgues J. \& Tram T. (2011). The Cosmic Linear Anisotropy Solving System (CLASS) II: approximation schemes. In J. Cosmology Astropart. Phys., vol. 1107, p. 034.

Bode P., Ostriker J.P. \& Turok N. (2001). Halo formation in warm dark matter models. In ApJ, vol. 556, pp. 93.

Boyanovsky D., de Vega H. \& Sanchez N. (2008). The dark matter transfer function: free streaming, particle statistics and memory of gravitational clustering. In Phys. Rev. D, vol. D78, p. 063546.

de Blok W.J.G. (2010). The Core-Cusp Problem. In Advances in Astronomy, vol. 2010, 789293.

Dunstan R.M., Abazajian K.N., Polisensky E. \& Ricotti M. (2011). The halo model of large scale structure for warm dark matter. In ArXiv e-prints.

Lesgourgues J. (2011). The Cosmic Linear Anisotropy Solving System (CLASS) III: Comparision with $C A M B$ for $\Lambda C D M$. In ArXiv e-prints.

Markovič K., Bridle S., Slosar A. \& Weller J. (2011). Constraining warm dark matter with cosmic shear power spectra. In J. Cosmology Astropart. Phys., vol. 1101, p. 022.

Polisensky E. \& Ricotti M., Constraints on the dark matter particle mass from the number of milky way satellites. In American Astronomical Society Meeting Abstracts \#215, vol. 42 of Bulletin of the American Astronomical Society, p. 408.02 (2010).

Schneider A., Smith R.E., Maccio A.V. \& Moore B. (Jul. 2012). Nonlinear Evolution of Cosmological Structures in Warm Dark Matter Models. In MNRAS, vol. 424, pp. 684.

Seljak U., Slosar A. \& McDonald P. (2006). Cosmological parameters from combining the Lyman$\alpha$ forest with CMB, galaxy clustering and SN constraints. In J. Cosmology Astropart. Phys., vol. 0610, p. 014. 
Semboloni E., Hoekstra H., Schaye J., van Daalen M.P. et al. (2011). Quantifying the effect of baryon physics on weak lensing tomography. In MNRAS, vol. 417, pp. 2020.

Sheth R.K. \& Tormen G. (1999). Large scale bias and the peak background split. In MNRAS, vol. 308, p. 119.

Simon J.D. \& Geha M. (Nov. 2007). The Kinematics of the Ultra-faint Milky Way Satellites: Solving the Missing Satellite Problem. In ApJ, vol. 670, pp. 313.

Smith R. et al. (2003). Stable clustering, the halo model and nonlinear cosmological power spectra. In MNRAS, vol. 341, p. 1311.

Smith R.E. \& Markovič K. (2011). Testing the warm dark matter paradigm with large-scale structures. In Phys. Rev. D, vol. D84, p. 063507.

Springel V. (2005). The cosmological simulation code GADGET-2. In MNRAS, vol. 364, pp. 1105. http://www.mpa-garching.mpg.de/gadget/.

van Daalen M.P., Schaye J., Booth C. \& Vecchia C.D. (2011). The effects of galaxy formation on the matter power spectrum: A challenge for precision cosmology. In MNRAS, vol. 415, pp. 3649 .

Viel M., Haehnelt M.G. \& Springel V. (2004). Inferring the dark matter power spectrum from the Lyman- $\alpha$ forest in high-resolution QSO absorption spectra. In MNRAS, vol. 354, p. 684.

Viel M., Lesgourgues J., Haehnelt M.G., Matarrese S. et al. (2005). Constraining warm dark matter candidates including sterile neutrinos and light gravitinos with WMAP and the Lyman- $\alpha$ forest. In Phys. Rev. D, vol. D71, p. 063534.

Viel M., Markovič K., Baldi M. \& Weller J. (2012). The Non-Linear Matter Power Spectrum in Warm Dark Matter Cosmologies. In MNRAS, vol. 421, pp. 50.

Wang J. \& White S.D. (2007). Discreteness effects in simulations of hot/warm dark matter. In MNRAS.

Zavala J., Jing Y., Faltenbacher A., Yepes G. et al. (2009). The velocity function in the local environment from $\Lambda C D M$ and $\Lambda W D M$ constrained simulations. In ApJ, vol. 700, pp. 1779.

Zavala J., Okamoto T. \& Frenk C.S. (2008). Bulges versus disks: The evolution of angular momentum in cosmological simulations of galaxy formation. In MNRAS, vol. 387, p. 364.

Zentner A.R. (2005). The Halo Model. In online. 